\title{
A Fatal Case of Severe Acute Organophosphorus Pesticide Poisoning Complicated with Secondary Hemophagocytic Lympho-Histiocytosis, Severe Lower Intestinal Hemorrhage and Intestinal Mucormycosis
}

\author{
Arun Agarwal, Chandra P. Tanwar, Yogesh K. Gupta, and Abhishek Chandra
}

\section{ABSTRACT}

A man in his 50's, under influence of alcohol, accidentally ingested a pesticide, and was referred for further management and admitted to our hospital. An empty can of Curacron ${ }^{\circledR}$ was found at the site in his farm where he took the alleged pesticide. This raised the suspicion of organophosphorus pesticide poisoning and he was managed at two medical centres before getting admitted to our hospital. His hospital course was complicated with multiorgan dysfunction, shock, respiratory failure and intermediate syndrome. On day five he developed secondary hemophagocytic lymphohistiocytosis (sHLH) and had hematochezia on day six. Colonoscopy revealed multiple circumferential ulcerations in descending and sigmoid colon with luminal narrowing. Biopsy of colonic tissue showed evidence of intestinal Mucormycosis. The clinical presentation of organophosphorus pesticide poisoning in this patient was complicated with multiple issues and included sHLH, chemical gastroenteritis, hemorrhagic ulcers and intestinal zygomycosis. The organophosphorus pesticide ingested by the patient was a $50 \%$ emulsifiable concentrate of profenofos along with vegetable oil, soyabean oil and polyglycol ether alkyl aryl sulphate calcium salt $5.25 \% \mathrm{w} / \mathrm{w}$ as an emulsifier/spreading agent. The management of the patient is discussed. Due to the possibility of the emulsifier adhering to the gastrointestinal tract and causing mucosal injury, it is necessary to identify the drug composition and ingredients of the pesticide as soon as possible when managing organophosphorus poisoning.

\footnotetext{
Keywords: Gastrointestinal bleeding, hemophagocytic lymphohistiocytosis, mucormycosis, organophosphorus pesticide poisoning, profenofos, spreading agent.
}

\section{INTRODUCTION}

Pesticides play an important role in the high productivity achieved in modern time's agriculture through the control of pests. Organophosphorous (OP) poisoning is one of the most common poisonings seen in India and is one of the important reasons for hospitals and intensive care units' admission in the developing countries [1]. OP compounds act through inhibition of enzyme acetylcholinesterase and are known to present with a complex of symptoms caused by additives (such as organic solvents, emulsifiers, and spreading agents), as well as neurological symptoms due to the effects of the main ingredient (organophosphate) [1], [2]. The symptoms of OP pesticide poisoning have been classified into three categories according to the timing of their appearance: acute, subacute, and delayed. There have been few reported cases of significant lower gastrointestinal bleeding following organophosphorus pesticide poisoning but not with sHLH
Published Online: December 13,2021

ISSN: $2736-5476$

DOI: 10.24018 /ejclinicmed.2021.2.6.152

\section{A. Agarwal*}

Department of Internal Medicine, Fortis Escorts Hospital, Jaipur, India.

(e-mail: mpicdrarun@gmail.com) C. P. Tanwar

Department of Gastrointestinal Medicine, Fortis Escorts Hospital, Jaipur, India.

(e-mail:

chandra.tanwar@fortishealthcare.com)

Y. K. Gupta

Department of Microbiology

Fortis Escorts Hospital, Jaipur, India.

(e-mail: dr.yogeshmicro@gmail.com)

A. Chandra

Department of Sports Medicine, All India Institute of Medical Sciences, Rishikesh, India. (e-mail:

abhishekchandra.dr@gmail.com)

*Corresponding Author and Intestinal Mucormycosis. Recently, we encountered a patient who developed sHLH and acute severe gastrointestinal bleeding after ingesting an organophosphorus pesticide. He also had intestinal Mucormycosis. He succumbed to severe OP poisoning and associated complications. We report this patient with a review of the literature as we could not find any other report in the literature of a case of OP poisoning complicated with sHLH, intestinal Mucormycosis and acute intestinal hemorrhage.

\section{CASE Report}

A 52-year-old farmer, chronic alcohol consumer with history of depression, was brought to the emergency department with a history of consumption of profenofos (CuracronR) mixed with alcohol (quantity unknown) around $9 \mathrm{pm}$ on 24/06/20. It was followed by repeated vomiting and difficulty in breathing. He took primary treatment locally 
where gastric lavage was done and he was referred to another health care centre where he was admitted and managed with Injection Atropine, PAM, Vasopressor (Noradrenaline) and was put on mechanical ventilation. He was referred to us on 26/06/2020 for further management.

On admission on 26/06/21 at Fortis Escorts Hospital, Jaipur, he was conscious, on vasopressor support, mechanical ventilation (CMV, TV $460 \mathrm{ml}$, PEEP $8 \mathrm{cms}$ of water, $\mathrm{FIO}_{2}$ $60 \%$, RR 20/minute), Glasgow coma score (GCS) of 6/15 (E2VTM4), pin-point pupils, copious oral secretions and was catheterized. His blood pressure was $100 / 69 \mathrm{~mm} \mathrm{Hg}$, pulse rate was $123 /$ minute, body temperature $1030 \mathrm{~F}$ and oxygen saturation was $100 \%$. Arterial blood gas showed mixed metabolic and respiratory acidosis $(\mathrm{pH} 7.307$, partial pressure of carbon dioxide ( $\mathrm{pCO} 2) 43.8$, serum bicarbonate $\left(\mathrm{HCO}_{3}\right)$ 21.4). Bilateral wheeze was heard on auscultation of the chest. Abdomen was soft and non-distended.

Since he received loading and subsequent doses of atropine and PAM outside from where he was referred, in view of severe poisoning and persistent sign and symptoms of intoxication, atropine was continued in infusion at $10 \mathrm{mg} / \mathrm{h}$ and Pralidoxime (PAM) was given in infusion at a rate of 500 $\mathrm{mg} / \mathrm{h}$ Serum Acetyl cholinesterase level was $<1 \mathrm{KU} / \mathrm{L}$ (reference range 4.62-11.5 KU/L).

Following admission to ICU, he was further evaluated and had multi organ dysfunction. His biochemistry and other investigations are tabulated in Table I. He had Hepatitis, Myocarditis with hypotension, acute kidney injury, sepsis, thrombocytopenia with cholinergic symptoms. His Procalcitonin was $100 \mathrm{ng} / \mathrm{ml}$, Trop T 1315 ng/ml, CK-MB $24.2 \mathrm{IU} / \mathrm{ml}$, Pro BNP > $35000 \mathrm{pg} / \mathrm{ml}$, and serum Amylase $1159 \mathrm{mg} / \mathrm{dl}$. His 2D echo showed Global LV hypokinesia with LVEF $40 \%$. He was managed with Inj. Atropine and PAM as per protocol, Torsemide, Noradrenaline support, antibiotics (Meropenem and Teicoplanin) and other supportive treatment. On 28/07/21 he developed leucopenia along with thrombocytopenia. On 29/06/20 he had melaena. Ultrasonography of abdomen showed mild hepatosplenomegaly. In view of persistent fever, multiple organ dysfunctions, bicytopenia, hepatosplenomegaly a possibility of sHLH (secondary hemophagocytic lymphohistiocytosis) was kept and he was further evaluated. His ferritin levels were very high (>39000) supporting diagnosis of sHLH. His $\mathrm{H}$ score was 234 points with a 98$99 \%$ probability of hemophagocytic syndrome. SLED (sustained low efficiency dialysis) was done in view of decrease in urine output and worsening azotemia. 2 Units PRBC (packed red blood cells) and 6U RDP (random donor platelets) were transfused on 30.06 .20 and upper GI endoscopy (UGIE) was planned and done. UGIE done on 30/06/21 showed erosive pan gastritis and duodenitis (Fig 1). He was managed with all supportive critical care. Atropine and PAM were stopped on 30/06/20. He was also given GCSF 600 ug IV for severe leucopenia and steroids pulse and IVIG (intravenous immunoglobulin) for sHLH (250 mg MPS daily for 3 days). His Natural Killer cell panel show low CD3 and CD (16+56). He had high EBV (NA) IgG and normal EBV (VCA) IgM. This ruled out acute EBV infection as cause for underlying sHLH syndrome. On 30/06/20 (Day 6 of poisoning) physical examination revealed dilated pupils (5 $\mathrm{mm}$ ), multiple cranial nerve palsies (III, IV, V, VI and VII cranial nerves bilaterally), weakness of neck flexors, flaccid extremities and decreased deep tendon reflexes. He was diagnosed to have developed intermediate syndrome. Mechanical ventilation was continued. Plasmapheresis (5 sessions) was done as there was no improvement in his clinical condition and persistently low serum AChE levels with fresh frozen plasma (FFP) as replacement fluid. Colonoscopy was done on $03 / 07 / 21$ for persistent melaena/hematochezia and it could be done only up to descending colon. It showed multiple circumferential superficial and deep ulcers with slough with oozing on descending colon, sigmoid colon, and rectum (Fig. 2). Biopsy was done which show broad, non-septate, ribbon like wide angle branching fungal hyphae suggestive of Mucormycosis (Fig 3). There was no evidence of Inflammatory Bowel disease or granulomas. His ET secretions also grew significant Candida albicans and he was given echinocandin -Micafungin. It was discussed with microbiology team and on 07/07/20 it was confirmed to be intestinal Mucormycosis. Micafungin was changed to liposomal Amphotericin B (LAmB). Tracheostomy was done on 07/07/20. He was maintained on mechanical ventilation, SLED, vasopressor support along with antibiotics, antifungal and supportive treatment but succumbed to his illness on 12/05/21.

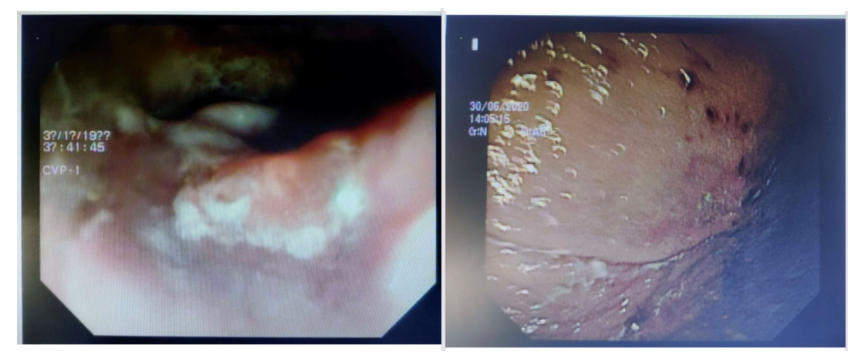

Fig. 1. (Left) Circumferential, irregular ulcerations with slough; (Right) UGIE: Multiple erosions in body of stomach.

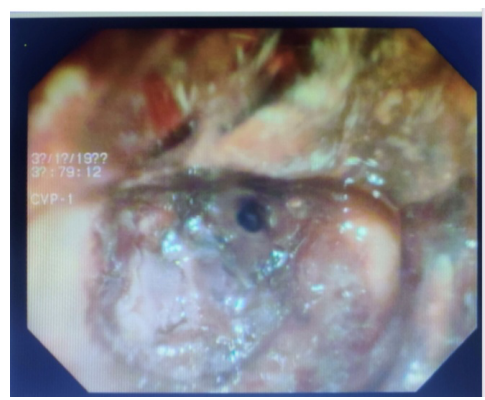

Fig. 2. Colonoscopy: Circumferential ulceration with narrowing of lumen, erosions and slough

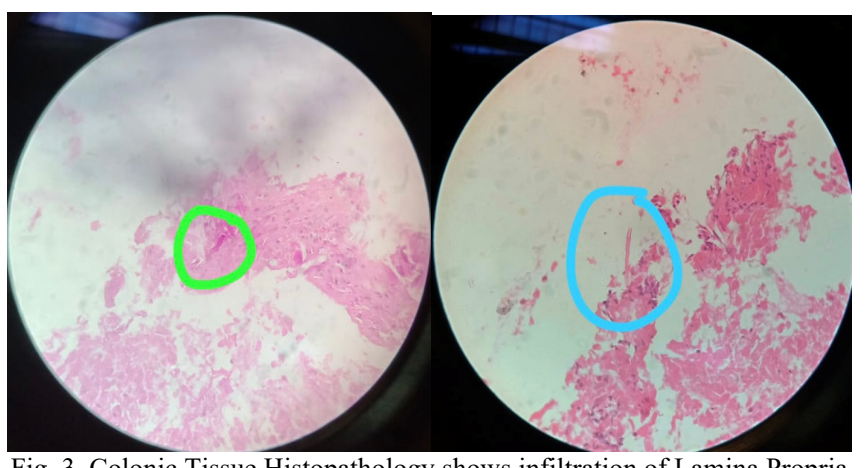

Fig. 3. Colonic Tissue Histopathology shows infiltration of Lamina Propria with Lymphocytes, few necrotic bits, foamy Macrophages and Fungal hyphae (Highlighted). Fungal hyphae are broad, non-septate, ribbon like with wide angle branching suggestive of Mucormycosis. 


\section{Discussion}

We have reported a case of severe fatal OP poisoning who developed sHLH syndrome, intestinal ulcers with lower GI bleed, intestinal Mucormycosis, and intermediate syndrome with multiple cranial nerve palsies and had persistently reduced plasma choline esterase (PCE) activity. To the best of our knowledge this is the first case report of severe OP poisoning who developed sHLH syndrome. The patient discussed was a farmer and had taken alcohol, some meals cooked in fields (possibly contaminated), and consumed unspecified amount of OP pesticide. As reported, by the time he was admitted with us he had already developed intermediate syndrome, was being mechanically ventilated and had developed multi organ dysfunction with shock.

OP compounds act through inhibition of enzyme acetylcholinesterase and several studies have found that estimation of plasma cholinesterase (PCE) levels on the day of presentation were reduced in almost all cases of OP poisoning [1], [3]. This patient had severely reduced PCE activity, and it did not recover till his demise despite management including fresh frozen plasma and Plasmapheresis. Estimation of PCE level at admission can be used to classify severity of OP poisoning, need of mechanical ventilation and to estimate its prognosis [1], [3], [4]. Further, periodic estimation of PCE levels could be more informative in predicting the prognosis or severity of poisoning and to monitor the effectiveness of therapeutic management [1].

Intermediate syndrome (IMS) in OP poisoning usually occurs 2-4 days after exposure when the symptoms and signs of the acute cholinergic syndrome abates. It typically affects conscious patients without cholinergic signs, and involve the muscles of respiration, proximal limb muscles, neck flexors, and muscles innervated by motor cranial nerves [5], [6]. Our patient developed IMS on Day 6 after exposure and has typical IMS features along with multiple cranial nerve palsies (III to VII). Intubation and mechanical ventilation were needed for respiratory failure
Gastrointestinal complications like lower gastrointestinal hemorrhage [2] mucosal erosions [7], gastric ulcers with perforation and peritonitis [8], duodenal ulcers [8] etc. have been reported in literature. Our patient also had erosive pan gastritis and duodenitis. Limited colonoscopy done showed multiple circumferential superficial and deep ulcers with slough and oozing in descending colon, sigmoid colon, and rectum. OP poisoning is known to cause vascular endothelial dysfunction, microvascular thrombosis leading to bowel wall ischemia, enteritis, ulcers, and perforation [2], [9], [10]. Other coingestants taken could also have contributed to the mucosal erosions. A further search revealed that he had consumed Curacron (trade name) and it contains $50 \%$ profenofos as the main ingredient, vegetable and soyabean oils as emulsifiers, an organic solvent and 5.25\% polyglycol ether alkyl aryl sulfonate calcium salt as a spreading agent. Since vegetable oils are unlikely to cause melena, the spreading agent used to stabilize profenofos could be a possible cause of mucosal injury [2]. The histopathology examination of the colonic biopsy showed presence of Mucormycosis. Intestinal Mucormycosis affecting an individual without the classic risk-factors is uncommon. In the present case, ingestion of contaminated food, a temporary suppression of the immune system due to secondary hemophagocytic lymphohistiocytosis syndrome (low natural killer cell levels), use of steroids to treat sHLH, prolonged hospitalization in multiple health care facilities could have predisposed to mycosis. Diagnosis of gastrointestinal Mucormycosis during life is very difficult as opposed to cutaneous and rhinocerebral forms. In a study on 129 cases of Mucormycosis, ante-mortem diagnosis of the gastrointestinal cases could not be done in any case whereas in the rhino-cerebral and cutaneous forms, ante-mortem diagnosis could be done in most of the cases ( $91 \%$ and $100 \%$, respectively) [11]. The present case is an exception as the diagnosis was made during the ante-mortem period even though the patient succumbed to the disease.

TABLE I: HEMATOLOGY, BIOCHEMISTRY AND OTHER INVESTIGATIONS

\begin{tabular}{|c|c|c|c|c|c|c|c|c|c|c|c|c|c|}
\hline & Normal values/Units & $26 / 6$ & $27 / 6$ & $28 / 6$ & $29 / 6$ & $30 / 6$ & $1 / 7$ & $2 / 7$ & $3 / 7$ & $4 / 7$ & $5 / 7$ & $6 / 7$ & $7 / 7$ \\
\hline Hemoglobin & $13.8-17.2 \mathrm{Gram} / \mathrm{dl}$ & 11.5 & 10.4 & 10.0 & 10.6 & 8.5 & 10.2 & 8.9 & 10.5 & 10.4 & & & \\
\hline $\begin{array}{c}\text { Total Leucocyte } \\
\text { count }\end{array}$ & $4-5-11 \times 103$ cells/ mm3 & 9.2 & 5.5 & 1.6 & 1.3 & 2.2 & 26.1 & 37.9 & 29.8 & 16.3 & & & \\
\hline Platelets & $150-450 \times 103 / \mathrm{mm} 3$ & & 88 & 50 & 45 & 60 & 65 & 75 & 50 & 35 & 45 & & \\
\hline Plasma & $4.62-11.5 \mathrm{KU} / \mathrm{L}$ & $<1.0$ & & $<1.0$ & & & & & $<1.0$ & & & & $<1.0$ \\
\hline \multicolumn{14}{|l|}{$\begin{array}{c}\text { Cholinesterase } \\
(4.62-11.5 \mathrm{KU} / \mathrm{L})\end{array}$} \\
\hline Creatinine & $0.9-1.3 \mathrm{mg} / \mathrm{dl}$ & 4.68 & 5.39 & 5.86 & 6.19 & 4.46 & & 3.62 & 4.64 & 4.25 & 4.27 & & \\
\hline Triglycerides & $<150 \mathrm{mg} / \mathrm{dl}$ & & & & & 138 & & & & & & & \\
\hline Procalcitonin & $<0.15 \mathrm{ng} / \mathrm{ml}$ & & $>100$ & & $>100$ & & & & & 11.81 & & & \\
\hline SGOT & $8-45 \mathrm{U} / \mathrm{L}$ & 1000 & & 472 & 608 & & & & 129 & & & & \\
\hline SGPT & $7-56 \mathrm{U} / \mathrm{L}$ & 621 & & 494 & 396 & & & & 51 & & & & \\
\hline $\mathrm{LDH}$ & $140-280 \mathrm{U} / \mathrm{L}$ & 1389 & & 1207 & & & 1180 & & 804 & & & & \\
\hline Amylase & $30-110 \mathrm{U} / \mathrm{L}$ & 1159 & & & & & & & & & & & \\
\hline Lipase & $10-140 \mathrm{U} / \mathrm{L}$ & 10 & & & & & & & & & & & \\
\hline TROP T & $0-0.4 \mathrm{ng} / \mathrm{ml}$ & & 1315 & & & & & & & & & & \\
\hline Pro BNP & $<125 \mathrm{pg} / \mathrm{ml}$ & & $>35000$ & & & & & & & & & & \\
\hline D-Dimer & $<500 \mathrm{ng} / \mathrm{ml}$ & & & & & & & & $>2,<4$ & & & & \\
\hline Fibrinogen & $200-400 \mathrm{mg} / \mathrm{dl}$ & & & & & & & & 715 & & & & \\
\hline Ferritin & $20-250 \mathrm{ng} / \mathrm{ml}$ & & & & & & 39464 & & & & & & \\
\hline NK Cell panel & $60-85 \%$ & & & & & & & & & & & & 44.6 \\
\hline $\mathrm{CD} 3, \mathrm{CD}(16+56)$ & $5-28 \%$ & & & & & & & & & & & & 1.6 \\
\hline CD45 & $0-99 \%$ & & & & & & & & & & & & 99 \\
\hline
\end{tabular}


Hemophagocytic lymphohistiocytosis (HLH) is a rare, under diagnosed, fatal and devastating hyper inflammatory syndrome that has gained increasing recognition over the past decade. Patients with HLH present with clinical and laboratory evidence of uncontrolled inflammation. It can be primary or secondary. We thought of this in the index case as he deteriorated rapidly, and his serum ferritin and NK cell panel were done. His serum ferritin was very high (39464 $\mathrm{ng} / \mathrm{ml})$ and CD $(16+56)$ cells were only $1.6 \%$. He fulfilled 5 of the 8 HLH 2004 diagnostic criteria criteria's [12]. The HScore can be used to estimate an individual's risk of having reactive hemophagocytic syndrome [13]. His H score was 234 points with a $98-99 \%$ probability of hemophagocytic syndrome. We could not find any other case of OP poisoning getting complicated with sHLH in literature. The trigger appears to be underlying infection of intestinal Mucormycosis and chest infection.

\section{CONCLUSION}

To conclude, we discussed a case of severe OP poisoning who got complicated with gastro-intestinal manifestations along with HLH syndrome and succumbed to his illness after a prolonged hospital stay. Serial measurements of PCE levels can be useful in predicting the length of hospital stay, duration of mechanical ventilation and the prognosis of the patient. Further, severe cases of OP poisoning can get complicated with involvement of gastro-intestinal system in the form of bleeding, ulcers, perforation, and even rare infections. Co-ingestants and the ingredients of the pesticide taken do have effects on morbidity and mortality.

\section{REFERENCES}

[1] Chaudhary SC, Singh K, Jain N, Vaish AK, Atam V, Patel ML et al Prognostic significance of estimation of pseudocholinesterase activity and role of pralidoxime therapy in organophosphorous poisoning. Toxicol Int. 2013; 20(3): 214-217.

[2] Tanabe K, Ikezaki T, Takano A, Suzuki T, Kitazawa H, Terasaki T. A case report of organophosphorus pesticide poisoning resulted in delayed severe lower intestinal hemorrhage. Science Postprint. 2013; 1(1): e00011.

[3] Areekul S, Srichairat S, Kirdudom P. Serum and red cell cholinesterase activity in people exposed to organophosphate insecticides. Southeast Asian J Trop Med Public Health. 1981; 12: 94-8.

[4] Goswamy R, Chaudhuri A, Mahashur AA. Study of respiratory failure in organophosphate and carbamate poisoning. Heart Lung. 1994; 23: 466-72.

[5] Karalliedde L, Baker D, Marrs T C. Organophosphate-induced intermediate syndrome: aetiology and relationships with myopathy. Toxicol Rev. 2006; 25(1): 1-14.

[6] Yang C, Deng JF. Intermediate syndrome following organophosphate insecticide poisoning. Journal of Chinese Medical Association. 2007; 70(11): 467-72.

[7] Tashev TS, Markov D. Stomach and duodenal lesions in patients with acute organophosphorus pesticide poisonings. V-treshni Boles. 1991; 30: 61-65.

[8] Tashev TS. 2 cases of severe gastroduodenal complications as a consequence of acute poisoning by organophosphorus pesticides. $V$ treshni Boles. 1988; 27: 136-39.

[9] Yoshida S, Okada H, Nakano S, Shirai K, Yuhara T, Kojima H, et al. Much caution does no harm! Organophosphate poisoning often causes pancreatitis. J Intensive Care. 2015; 3: 21.

[10] Mahajan RK, Rajan SJ, Peter JV, Suryawanshi MK. Intestinal perforation in organophosphorous poisoning: a case report. Journal of Clinical and Diagnostic Research. 2016; 10(3): GD06-GD07.

[11] Chakrabarti A, Das A, Sharma A, Panda N, Das S, Gupta KL, et al. Ten years' experience in zygomycosis at a tertiary care centre in India. $J$ Infect. 2001; 42: 261-6.
[12] Henter JI, Horne A, Aricó M, Egeler RM, Filipovich AH, Imashuku S, et al. HLH-2004: Diagnostic and therapeutic guidelines for hemophagocytic lymphohistiocytosis. Pediatr Blood Cancer. 2007; 48(2): 124-31.

[13] Fardet L, Galicier L, Lambotte O, Marzac C, Aumont C, Chahwan D, et al. Development and validation of the HScore, a score for the diagnosis of reactive hemophagocytic syndrome. Arthritis Rheumatol. 2014; 66(9): 2613-20.

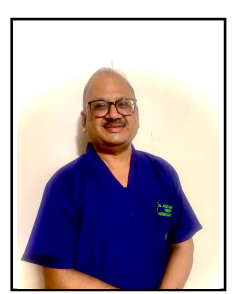

A. Agarwal, born on December 14, 1961, in Jaipur, India completed his MBBS and MD from the prestigious SMS Medical College in Jaipur. He completed his MD in 1989 and then joined Sir Gangaram Hospital and Hindu Rao Hospital in New Delhi, India for residency program.

He later started working as an independent consultant in Internal medicine with a keen interest in tropical and critical care medicine. He has about 50 peer reviewed publication in national and international journals. He is currently working as Director in the department of Internal Medicine at Fortis Escorts Hospital, Jaipur, India and is involved in teaching DNB students enrolled in the institute.

Dr Agarwal is a member of several prestigious societies including API, CSI, ISCCM and RSSDI. He has been awarded Dr JC Patel best paper award in 2016 for his work on hemophagocytic lymphohistiocytosis. 